\title{
Both AA genotype of rs165599 and Val/Val genotype of rs4680 in COMT are associated with an increased risk of recurrence of prostate cancer
}

\author{
Shixiong Yang ${ }^{1}$, Handong Fư ${ }^{1}$, Xiaobing Liu $^{2}$, Kun Yang ${ }^{3}$
}

\begin{abstract}
${ }^{1}$ Central Laboratory, Xiaogan Hospital Affiliated with Wuhan University of Science and Technology, Xiaogan Central Hospital, Xiaogan, China 2Department of Urology, Xiaogan Hospital Affiliated with Wuhan University of Science and Technology, Xiaogan Central Hospital, Xiaogan, China

${ }^{3}$ Department of General Surgery, Xiaogan Hospital Affiliated with Wuhan University

of Science and Technology, Xiaogan Central Hospital, Xiaogan, China
\end{abstract}

Submitted: 3 May 2020; Accepted: 7 November 2020

Online publication: 5 May 2021

Arch Med Sci

DOI: https://doi.org/10.5114/aoms/130290

Copyright $\odot 2022$ Termedia \& Banach

\section{Abstract}

Introduction: The catechol-O-methyltransferase (COMT) gene expression was reported to be associated with the recurrence of prostate cancer. Furthermore, it has been shown that rs165599 SNP in the 3'UTR of COMT affects the interaction between miR-138 and COMT, while another polymorphism (rs4680) located in the gene of COMT also affects its expression. Here, we explored the combined effect of rs165599 and rs4680 on the recurrence of prostate cancer.

Material and methods: Kaplan-Meier analysis was performed to analyse the PSA-progression-free survival of prostate cancer patients carrying different genotypes of rs 165599 and rs4680 SNPs. IHC and Western blot were used to evaluate the expression of COMT. Real-time PCR was carried out to examine the expression of miR-138 and COMT mRNA. Luciferase assay was performed to explore the inhibitory role of miR-138 in the expression of COMT carrying different genotypes.

Results: The PSA-progression-free survival was significantly increased in prostate cancer patients carrying the rs165599-AA + rs4680-Val/Val genotype. In concordance with this, the expression of COMT was remarkably elevated in the tissue samples collected from prostate cancer patients carrying the rs165599-AA + rs4680-Val/Val genotype. Luciferase assay demonstrated that COMT-G at rs 165599 was effectively suppressed by miR-138 through binding to the 3' UTR of COMT-G. Furthermore, COMT expression was notably suppressed by miR-138 precursors in primary prostate cancer cells carrying GG genotype at rs165599.

Conclusions: The findings of this study demonstrated that the carriers of AA genotype of rs165599 and Val/Val genotype of rs4680 showed an increased risk of prostate cancer recurrence, and these 2 polymorphisms may jointly function as a novel biomarker for the prognosis of prostate cancer recurrence.

Key words: prostate cancer, COMT, miRNA, rs165599, rs4680, recurrence in prostate cancer.

\section{Introduction}

Pancreatic cancer (PC) is the $7^{\text {th }}$ leading contributor for cancer fatalities in China and the $4^{\text {th }}$ leading contributor for cancer fatalities in the

\author{
Corresponding author: \\ Handong Fu \\ Central Laboratory Xiaogan \\ Hospital \\ Affiliated with Wuhan \\ University of Science and \\ Technology \\ Xiaogan Central Hospital \\ No. 6 Square Street \\ Xiaonan District, Xiaogan, \\ China, 432000 \\ Phone: 86-712-2857130 \\ E-mail: uro_bladder@yeah. \\ net
}


USA [1, 2]. In the USA alone, more than 250,000 PC patients die annually. As a result of the fact that little improvement has actually been made in the diagnosis as well as surgical procedures in the treatment of $P C$ in recent years, the prognosis of PC patients is the worst among all kinds of cancers $[3,4]$. It is vital to find unique approaches to address the conditions of individual PC patients.

The catechol-O-methyltransferase (COMT) gene is found on chromosome 22q11.2 [5]. There are actually 2 forms of COMT proteins translated from COMT: one is soluble and the other is bound on cell membrane [6]. COMT is an enzyme associated with the inactivation of catecholamines as well as catechol oestrogens, which have been demonstrated to possess carcinogenetic properties along with the capacity for DNA damage and induction of PC in rats [7]. Meanwhile, COMT can catalyse the O-methylation of catechol oestrogen 2-hydroxyestradiol to produce 2-methoxyestradiol, which has anti-angiogenic and anti-proliferative properties [8]. A typical polymorphism in COMT is a $\mathrm{G}$ to A mutation that takes place at codon 158 , which is also called Val158 Metorrs4680. The replacement of $G$ for $A$ causes the substitute of valine by methionine as well as 3- to 4-fold reduction in COMT activity. The Val/ Val phenotype (or G/G genotype) of COMT has been shown to be more active and stable as compared with the Met/Met phenotype (or A/A genotype). Many studies have performed genome wide screening without pinpointing the genres of COMT with considerable prospect for suicidal behaviours, with a few exceptions [9-12].

MicroRNA (miRNA) makes up a family of non-coding RNAs that are less than 23 nucleotides in size and can control the endogenous expression of genes through target mRNA degradation and translational inhibition [13]. In the last few years, miRNAs have been demonstrated to regulate several biological activities, such as cell growth, neuron distinction, and apoptosis [14-16]. Significantly, growing evidence indicates that miRNA expression might be related to cancer cell growth in PC patients [14, 17, 18]. Amongst many miRNAs, miR-138 has recently been shown as a significant tumour suppressor in various cancers, including osteosarcoma, cervical cancer, ovarian cancer, non-small cell lung cancer, and gallbladder cancer [19-21]. Primarily, miR-138 prevents the spread of cancer cells and causes their apoptosis while boosting their chemo sensitivity [22-24]. It was discovered that down-regulation of miR-138-5p was linked to autophagy in PC cells. Nevertheless, elevated miR-138-5p expression prevented the activation of autophagy dependent on the SIRT1/ FoxO1/Rab7 signalling. Also, SIRT1 might repress the growth of PC cells, making miR-138-5p a possible target in PC treatment [25].
It has been shown that COMT expression is associated with prostate cancer recurrence [26]. Furthermore, it has been shown that rs 165599 in the 3' untranslated region (3'UTR) of COMT affects the interaction between miR-138 and COMT, and another polymorphism ( $r$ 4680) located in the gene of COMT also affects the expression level of COMT [27-30]. Moreover, polymorphisms located in oestrogen metabolism-related genes, such as the AA genotype and the A allele of rs4680 in COMT, have been proven to be inversely associated with the risk of prostate cancer in Afro-Caribbean and native African men [31]. In this study, we hypothesized that the genotype difference of rs165599 and rs4680 is associated with the risk of prostate cancer recurrence and the PSA-progression-free survival period. To study the effect of genotype difference on prostate cancer recurrence, we studied the combined effect of both rs165599 and rs4680 single nucleotide polymorphisms (SNPs) on the recurrence of prostate cancer.

\section{Material and methods}

\section{Human subjects and sample collection}

In this work, to study the combined effect of rs165599 and rs4680 SNPs on prostate cancer, we recruited 224 prostate cancer patients and then partitioned them into 4 groups according to their genotypes of rs165599 and rs4680 SNPs: (1) rs165599-AA + rs4680-Val/Val group $(N=62) ;(2)$ rs165599-AA + rs4680- (Val/Met + Met/Met) group $(N=55)$; (3) rs165599- (GG+GA) + rs4680-Val/ Val group $(N=58)$; and (4) rs165599-(GG+GA) + rs4680- (Val/Met + Met/Met) group $(N=49)$. The information of all participants, including their age, tumour status, and Gleason score, was collected and compared among the 4 groups. Then, one-way analysis of variance (ANOVA) was utilized to perform the statistical comparison among the above 4 groups. In the next step, tissue and peripheral blood samples were collected from all subjects for the analyses described below. The institutional ethical committee approved the protocol of this study.

\section{Genotyping by direct sequencing}

Collected tissue and peripheral blood samples were treated with a GoldMag Mini Purification assay kit (GoldMag Co. Ltd., Xi'an, China), in accordance with the specific assay protocol provided by the assay kit manufacturer, to extract Genomic DNA content. Then, the quality and the concentration of each isolated DNA sample were assessed by using a Nanodrop 3000 spectrophotometer (Thermo Fisher Scientific, Waltham, MA) in accordance with the specific operating protocol provided by the instrument manufacturer. In the next step, the genotypes of rs 165599 and rs4680 SNPs 
were measured by using a multiplexed SNP Mass EXTEND assay designed with the Agena Mass ARRAY version 3.0 software (Agena Bioscience, San Diego, CA) in accordance with the specific instructions provided by the software vendor. The multiplexed SNP Mass EXTEND assay was carried out using an Agena Mass ARRAY RS1000 system in accordance with the specific operating protocol provided by the instrument manufacturer.

\section{RNA isolation and real-time PCR}

In order to explore the effect of rs165599 and rs4680 polymorphisms on the expression of their host gene COMT, we carried out qPCR and ELISA to analyse the expression of COMT in tissue samples of prostate cancer as well as peripheral blood samples collected from all prostate cancer patients carrying different genotypes. We also performed qPCR to examine the expression of miR-138 in these tissue and peripheral blood samples. To carry out the measurements, the total RNA content was first isolated from each sample by utilizing an RNAiso Plus RNA extraction reagent (Takara, Tokyo, Japan) in accordance with the specific protocol provided by the reagent manufacturer. Then, the isolated RNA from each sample was reverse transcribed into cDNA templates using a Taqman Advanced cDNA Synthesis kit (Thermo Fisher Scientific, Waltham, MA) and a Reverse Transcription kit (Applied Biosystems, Foster City, CA) in accordance with the specific assay protocols provided by the assay kit manufacturers. Then, quantitative real-time PCR was performed by making use of a Fast-Start Universal SYBR Green Master Mix assay kit (Roche, Basel, Switzerland) in accordance with the specific assay protocol provided by the assay kit manufacturer. The real-time PCR reaction was done on an ABI Prism $7900 \mathrm{HT}$ real-time PCR machine (Applied Biosystems, Foster City, CA) using $U 6$ and GAPDH as the internal controls for the normalization of measured relative expression of target genes, i.e. COMT mRNA and miR-138.

\section{Cell culture and transfection}

Primary prostate cancer cells were bought from the Shanghai Cell Bank at the Chinese Academy of Sciences (Shanghai, China). The cells were cultured in a RPMI 1640 medium added with $10 \%$ of foetal bovine serum (Gibco, Thermo Fisher Scientific, Waltham, MA) and suitable antibiotics. The culture conditions were saturated humidity at $37^{\circ} \mathrm{C}$ in $5 \%$ carbon dioxide. To further explore the regulatory network of rs165599 polymorphisms, miR-138 and COMT, we cultured primary prostate cancer cells carrying the GG and AA genotypes, and then transfected them with COMT siRNA and miR-138 precursors, respectively. In brief, the primary prostate cancer cells carrying the GG genotype of the rs165599 SNP were divided into 3 groups: (1) Scramble control group (primary prostate cancer cells treated with $50 \mathrm{nM}$ of a scramble control siRNA); (2) COMT siRNA group (primary prostate cancer cells treated with $50 \mathrm{nM}$ of COMT SiRNA); and (3) miR-138 precursor group (primary prostate cancer cells treated with $50 \mathrm{nM}$ of miR-138 precursors). Similarly, the primary prostate cancer cells carrying the AA genotype of the rs165599 SNP were also divided into 3 groups, i.e. (1) Scramble control group (primary prostate cancer cells treated with $50 \mathrm{nM}$ of a scramble control siRNA); (2) COMT SiRNA group (primary prostate cancer cells treated with 50 nM of COMT siRNA); and (3) miR-138 precursor group (primary prostate cancer cells treated with $50 \mathrm{nM}$ of miR-138 precursors). In the next step, the cells were transfected with corresponding siRNA/miRNA by using Lipofectamine 3000 (Invitrogen, Carlsbad, CA) in accordance with the specific transfection protocol provided by the manufacturer of the transfection reagent. At 48 hours post transfection, the cells were harvested for the analysis of target gene expression.

\section{Vector construction, mutagenesis, and luciferase assay}

Our sequence analysis showed that miR-138 could potentially bind to the 3' UTR of COMT. In order to examine whether rs 165599 polymorphisms affect the expression of COMT, the 3' UTR of COMT carrying the $A$ and $G$ allele of the rs165599 polymorphism was cloned into pcDNA luciferase vectors (Promega, Madison, WI), respectively, to establish COMT-G and COMT-A vectors, which were then transfected into PC3 cells along with miR-138 mimics by using Lipofectamine 3000 in accordance with the specific transfection protocol provided by the manufacturer of the transfection reagent. At 48 hours post transfection, the luciferase activity of transfected cells was assayed by using a Dual Luciferase reporter gene assay kit (Promega, Madison, $\mathrm{WI}$ ) in accordance with the specific assay protocol provided by the manufacturer of the assay kit.

\section{Western blot analysis}

The total protein content was extracted from each sample by making use of a radio immunoprecipitation assay kit (Solarbio Science \& Technology Co. Ltd., Beijing, China) in accordance with the specific assay protocol provided by the assay kit manufacturer. Then, $50 \mu \mathrm{g}$ of each protein sample was resolved by using SDS-PAGE and blotted onto polyvinylidene fluoride (PVDF) membranes, which was then blocked by utilizing $5 \%$ skimmed milk at ambient temperature for 1 hour before they were incubated for 24 hours at $4^{\circ} \mathrm{C}$ 
with anti-COMT primary antibody (Abcam, Cambridge, MA) followed by incubation with suitable HRP-conjugated secondary antibodies for 1 hour at room temperature in accordance with the specific instructions provided by the antibody manufacturer. After colour development by utilizing an enhanced chemiluminescence (ECL) assay kit (Amersham Pharmacia, Piscataway, NJ) in accordance with the specific assay protocol provided by the assay kit manufacturer, the relative protein expression of COMT in each sample was determined by means of a Bio-Rad imaging system (Bio-Rad Laboratories, Hercules, CA) in accordance with the instructions provided by the machine manufacturer.

\section{Immunohistochemistry (IHC)}

In order to explore the effect of rs165599 and rs4680 polymorphisms on the expression of their host gene COMT, we collected prostate cancer tissue samples from all patients and performed immunohistochemistry to evaluate COMT expression in each sample. In brief, the collected prostate cancer tissue samples were embedded in paraffin, fixed, deparaffinized, made transparent, and blocked with $5 \%$ goat serum before they were treated with anti-COMT primary antibodies and matching Alexa Fluor 488-labelled secondary antibodies in sequence (Santa Cruz Biotechnology, Santa (ruz, CA) in accordance with the instructions provided by the antibody manufacturer. After counterstaining with DAPI, the positive expression of COMT in each sample was evaluated by using a confocal microscope.

\section{ELISA}

The COMT activity in the collected peripheral blood samples was measured using a commercial ELISA assay kit (Thermo Fisher Scientific, Waltham,
$M A)$ in accordance with the specific assay protocol provided by the assay kit manufacturer.

\section{Statistical analysis}

Data analysis was carried out using SPSS 19.0 software (SPSS, Chicago, IL). The Kaplan-Meier method was used to compare the prostate-specific antigen (PSA)-progression-free survival of patients in the 4 groups. Comparisons between 2 groups was done by using Student's $t$ tests. The results are presented as mean \pm standard deviation. One-way ANOVA was used to compare multiple groups. A $p$ value of $<0.05$ was deemed statistically significant.

\section{Results}

Patients with rs165599-AA + rs4680-Val/ Val genotypes had the highest PSA-progression-free survival

In this work, we recruited 224 prostate cancer patients and partitioned them into 4 groups according to their genotypes at rs165599 and rs4680 SNP. The information of participants including their age, tumour status, and Gleason score was collected and is shown in Table I. ANOVA was utilized to perform the statistical comparison among the above 4 groups, and the results revealed that there is no obvious difference in all characters among the groups. The Kaplan-Meier method was used to compare the PSA-progression-free survival of patients in the 4 groups. The PSA-progression-free survival was highest in patients carrying the rs165599-AA + rs4680-Val/Val genotype, and it was lowest in patients carrying the rs165599$(\mathrm{GG}+\mathrm{GA})+$ rs4680- (Val/Met + Met/Met) genotype. The PSA-progression-free survival of patients carrying the rs165599-AA + rs4680- (Val/Met + Met/ Met) and rs165599- (GG+GA) + rs4680-Val/Val genotypes was moderate (Figure 1).

Table I. Basic information of participants grouped according to their genotypes

\begin{tabular}{|c|c|c|c|c|c|}
\hline $\begin{array}{l}\text { Character- } \\
\text { istics }\end{array}$ & $\begin{array}{l}\text { Rs165599-AA + } \\
\text { Rs4680-Val/Val } \\
\quad(N=62)\end{array}$ & $\begin{array}{c}\text { Rs165599-AA + } \\
\text { Rs4680- }(\text { Val/Met + } \\
\text { Met/Met })(N=55)\end{array}$ & $\begin{array}{c}\text { Rs165599- }(\mathrm{GG}+\mathrm{GA})+ \\
\text { Rs4680-Val/Val } \\
(N=58)\end{array}$ & $\begin{array}{c}\text { Rs165599-(GG+GA) + } \\
\text { Rs4680- }(\mathrm{Val} / \mathrm{Met}+ \\
\text { Met/Met) }(N=49)\end{array}$ & $P$ value \\
\hline Age [years] & $73.4 \pm 5.4$ & $70.6 \pm 5.6$ & $74.0 \pm 5.8$ & $71.2 \pm 5.1$ & 0.772 \\
\hline $\begin{array}{l}\text { Tumour } \\
\text { status }\end{array}$ & & & & & 0.632 \\
\hline T1 & $1(1.6)$ & $1(1.8)$ & $2(3.4)$ & $1(2.0)$ & \\
\hline $\mathrm{T} 2$ & $10(16.1)$ & $11(20.0)$ & $8(13.8)$ & $8(16.3)$ & \\
\hline T3 & $33(53.2)$ & $28(50.9)$ & $30(51.7)$ & $28(57.1)$ & \\
\hline T4 & $18(29.1)$ & $15(27.3)$ & $18(31.1)$ & $12(24.6)$ & \\
\hline $\begin{array}{l}\text { Gleason } \\
\text { score }\end{array}$ & & & & & 0.717 \\
\hline $2-7$ & $32(51.6)$ & $30(54.5)$ & $30(51.7)$ & $22(44.9)$ & \\
\hline $8-10$ & $30(48.4)$ & $25(45.5)$ & $28(48.3)$ & 27 (55.1) & \\
\hline
\end{tabular}


The expression of COMT in tissue samples and peripheral blood was remarkably elevated in patients carrying the rs165599-AA + rs4680-Val/Val genotype

We performed immunohistochemistry to evaluate COMT expression in various samples. The expression of COMT was significantly higher in tissue samples collected from patients carrying the rs165599-AA + rs4680-Val/Val genotype, and it was remarkably lower in tissue samples collected from patients carrying the rs165599-(GG+GA) + rs4680- (Val/Met + Met/Met) genotype (Figure 2). Moreover, we carried out qPCR and ELISA to analyse the expression of COMT in tissue samples and peripheral blood. The expression of COMT mRNA in tissue samples (Figure $3 \mathrm{~A}$ ) and peripheral blood (Figure $3 \mathrm{~B}$ ) was in good agreement with that revealed by IHC analysis.

MiR-138 inhibited the expression of COMT carrying the rs $165599-\mathrm{G}$ genotype by binding to the 3' UTR of COMT

No obvious difference was observed for the expression of miR-138 in either tissue samples (Fig-

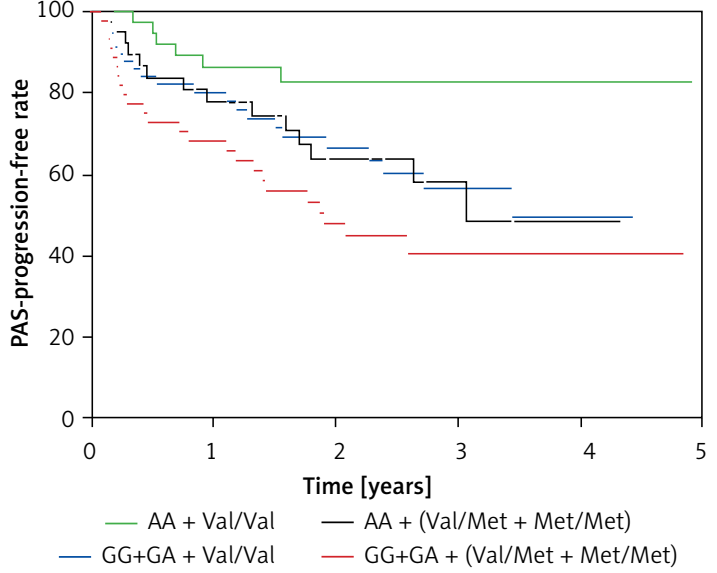

Figure 1. The PSA-progression-free survival was significantly higher in patients carrying the rs165599$\mathrm{AA}+\mathrm{rs} 4680-\mathrm{Val} / \mathrm{Val}$ genotype and lower in patients carrying the rs165599- $(\mathrm{GG}+\mathrm{GA})+\mathrm{rs} 4680-(\mathrm{Val} / \mathrm{Met}+$ Met/Met) genotype

ure 4 A) or peripheral blood (Figure 4 B). Sequence analysis showed that miR-138 could potentially bind to the 3' UTR of COMT (Figure $5 \mathrm{~A}$ ). In order to examine whether rs 165599 polymorphisms affect the expression of COMT, luciferase vectors
$\mathrm{AA}+\mathrm{Val} / \mathrm{Val}$

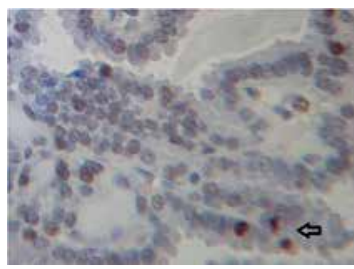

$\mathrm{AA}+(\mathrm{Val} / \mathrm{Met}+$ Met/Met $)$

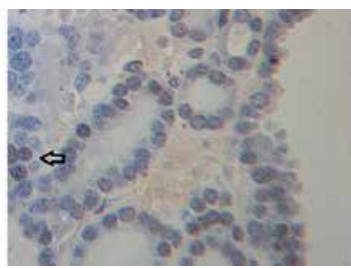

$\mathrm{GG}+\mathrm{GA}+\mathrm{Val} / \mathrm{Val}$

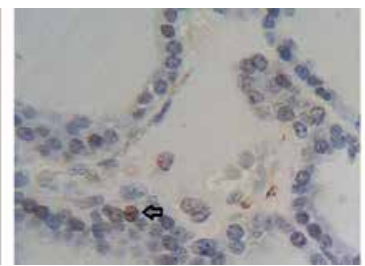

$\mathrm{GG}+\mathrm{GA}+($ Val/Met + Met/Met $)$

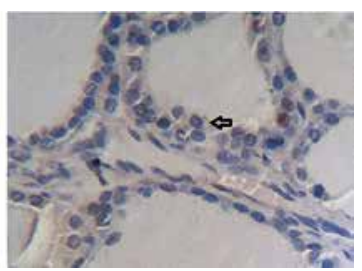

Figure 2. Immunohistochemistry analysis showed that the expression of COMT protein was increased in the tissue samples collected from patients carrying the rs165599-AA + rs4680-Val/Val genotype and decreased in tissue samples collected from patients carrying the rs165599-(GG+GA) + rs4680- (Val/Met + Met/Met) genotype

A

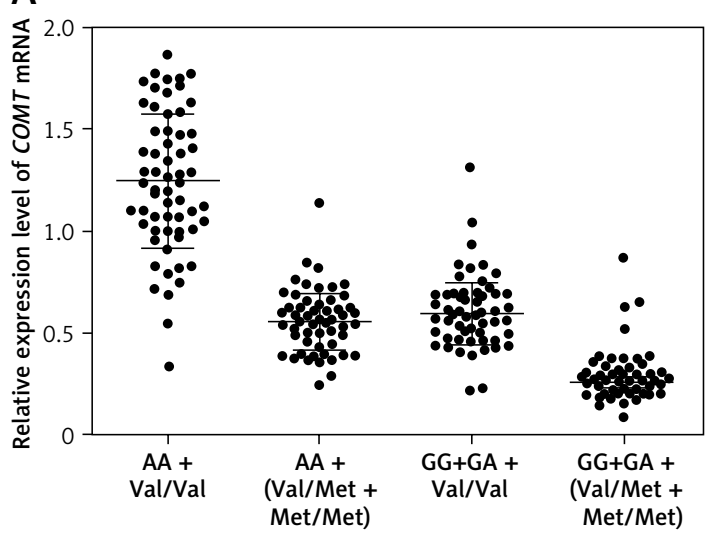

B

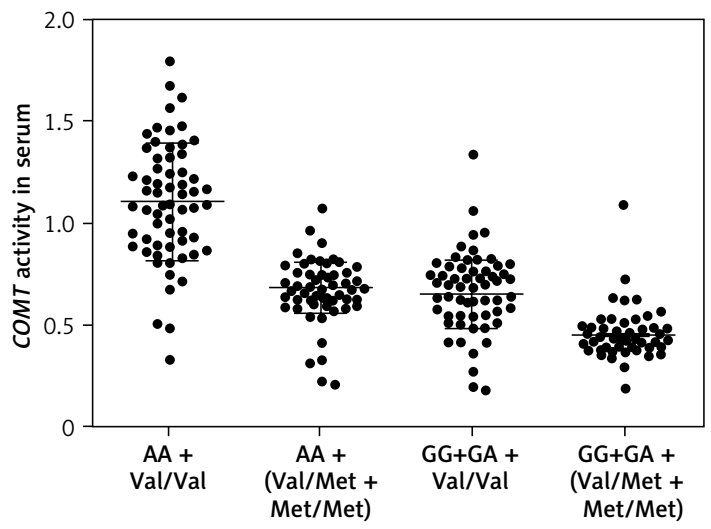

Figure 3. The expression and activity of COMT were increased in patients carrying the rs165599-AA + rs4680-Val/ Val genotype and decreased in patients carrying the rs165599-(GG+GA) + rs4680- (Val/Met + Met/Met) genotype. A - QPCR analysis showed that the expression of COMT mRNA was increased in the tissue samples collected from patients carrying the rs165599-AA + rs4680-Val/Val genotype and decreased in the tissue samples collected from patients carrying the rs165599-(GG+GA) + rs4680- (Val/Met + Met/Met) genotype. B - ELISA analysis showed that the activity of COMT was increased in the serum collected from patients carrying the rs165599-AA + rs4680-Val/ $\mathrm{Val}$ genotype and decreased in the serum collected from patients carrying the rs165599- $(\mathrm{GG}+\mathrm{GA})+\mathrm{rs} 4680-(\mathrm{Val} /$ Met + Met/Met) genotype 
A

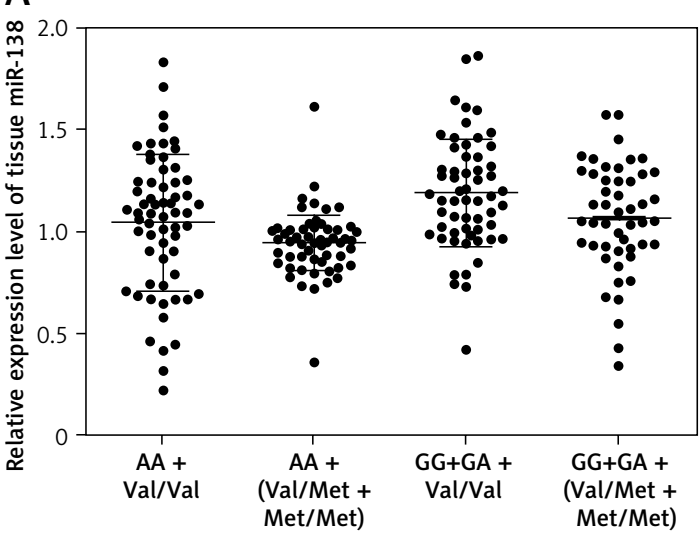

B

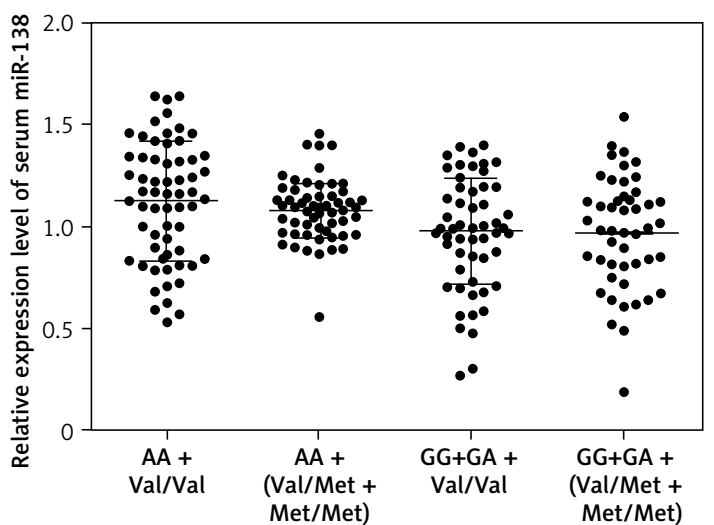

Figure 4. No obvious difference was observed for the expression of miR-138 in the tissue samples and serum collected from patients carrying different genotypes. A - No apparent difference was observed for the expression of miR-138 in the tissue samples collected from patients carrying different genotypes. B - No apparent difference was observed for the expression of miR-138 in the serum collected from patients carrying different genotypes

carrying COMT-G and COMT-A were established and transfected into PC3 cells along with miR-138 mimics. The luciferase activity of COMT-G vector was significantly suppressed by miR-138 mimics, whereas no apparent suppression was observed for COMT-A vector (Figure $5 \mathrm{~B}$ ).

\section{MiR-138 precursors effectively suppressed} the expression of COMT in primary prostate cancer cells carrying the GG genotype

To further explore the regulatory network of rs165599 polymorphisms, miR-138 and COMT, we cultured primary prostate cancer cells carrying the GG and AA genotypes, and then transfected them with COMT SiRNA and miR-138 precursors, respectively. The dramatically elevated expression of miR-138 in primary prostate cancer cells carrying the GG (Figure $6 \mathrm{~A}$ ) and AA (Figure $7 \mathrm{~A}$ ) genotypes indicated successful transfection of miR-138 precursors. QPCR and Western blot analysis showed that the expression of COMT mRNA (Figure $6 \mathrm{~B}$ ) and protein (Figure $6 \mathrm{C}$ ) was notably decreased in primary prostate cancer cells carrying the GG genotype after transfection with miR-138 precursors. The inhibitory effect of miR-138 precursors on the expression of COMT in primary prostate cancer cells carrying the GG genotype was even stronger than that of COMT SiRNA. In addition, the expression of COMT mRNA (Figure $7 \mathrm{~B}$ ) and protein (Figure $7 \mathrm{C}$ ) was decreased to a lesser extent in primary prostate cancer cells carrying the AA genotype after transfection with miR-138 precursors.

\section{Discussion}

The findings of this study support our hypothesis that the genotypes of rs165599 and rs4680
A

hsa-miR-138/rs165599(A)

miR-138 3' GCCGG----ACUAAGUGUU G-U GGUCGA 5'

COM_A

5' AGCCCCAUGGGG--ACGACUGCCAGCC 3'

hsa-miR-138/rs165599(G)

miR-138

COM_G
B

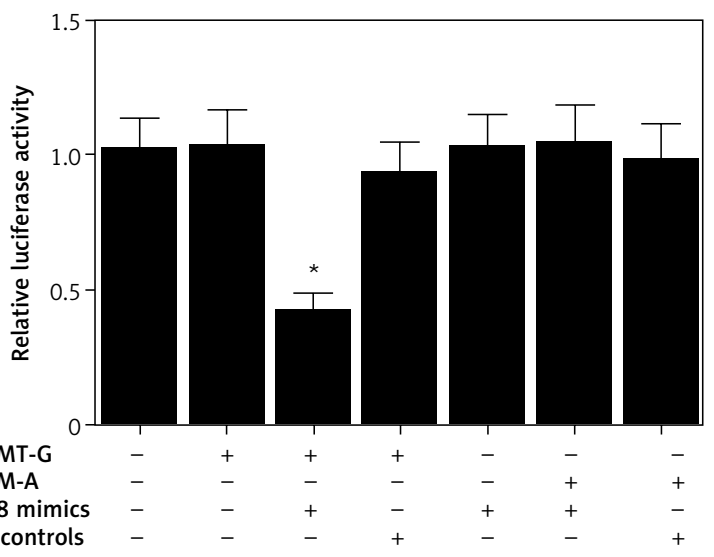

Figure 5. The luciferase activity of COMT-G was inhibited by miR-138. A - Sequence analysis showed that miR-138 could potentially bind to COMT carrying the G allele of rs 165599 SNP. B - The luciferase activity of COMT-G was suppressed by miR-138 in PC3 cells ( ${ }^{*} P$ value $<0.05$ vs. miRNA controls) 
A
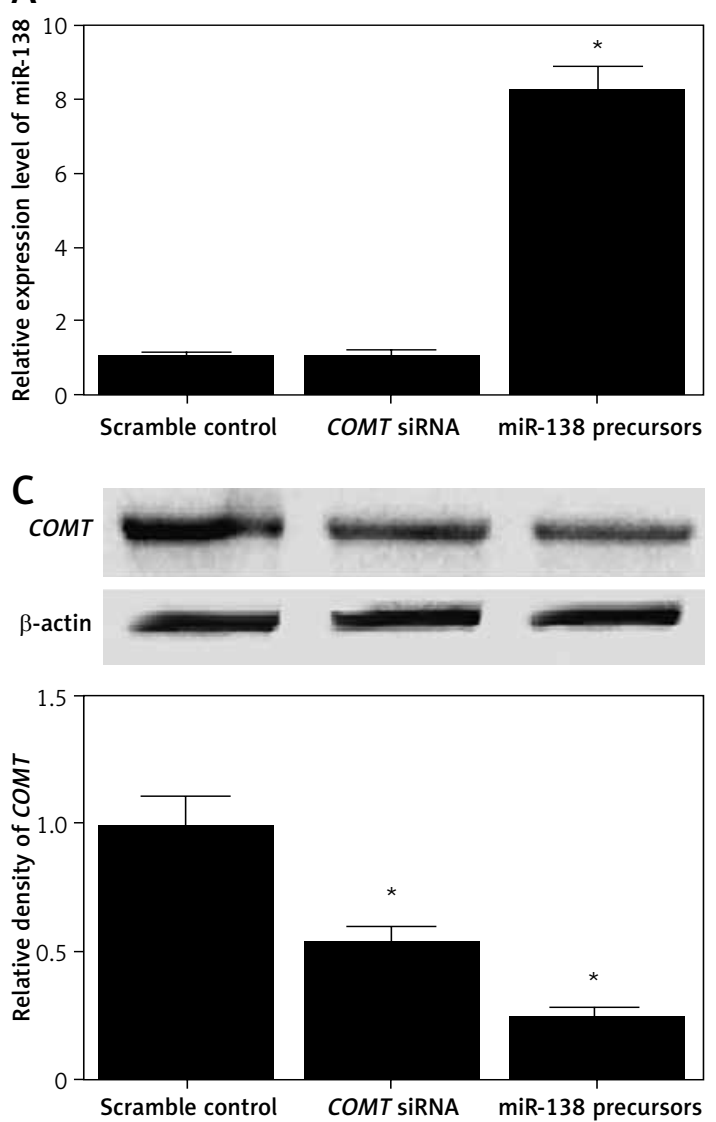

A

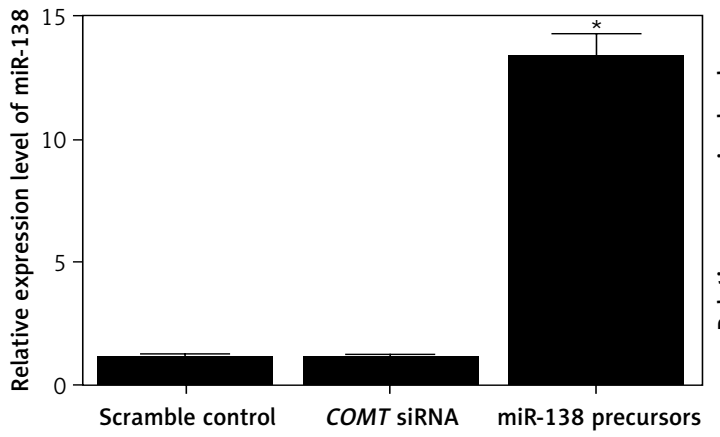

C
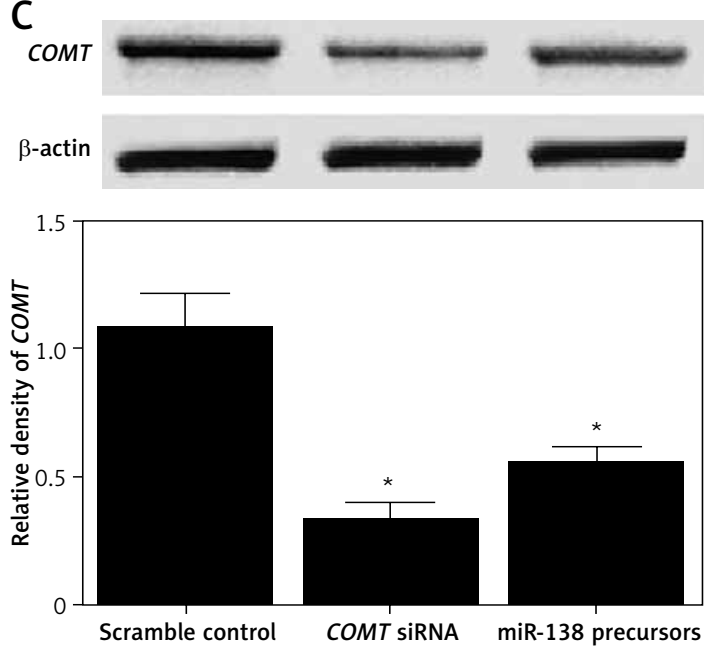

B

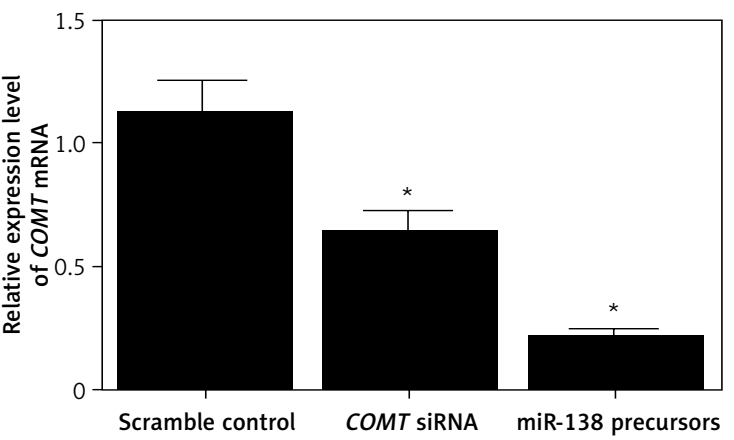

Figure 6. MiR-138 precursors effectively suppressed the expression of COMT in primary prostate cancer cells carrying the GG genotype. A - Dramatic elevation of miR-138 expression indicated efficient transfection of miR-138 precursors into primary prostate cancer cells $\left({ }^{*} p<0.05\right.$ vs. scramble control). B - The inhibition of COMT mRNA expression by miR-138 precursors was stronger than that by COMT SiRNA in primary prostate cancer cells carrying the GG genotype ( ${ }^{*} p<0.05$ vs. scramble control). $\mathbf{C}-$ The inhibition of COMT protein expression by miR-138 precursors was stronger than that by COMT siRNA in primary prostate cancer cells carrying the GG genotype $\left({ }^{*} p<0.05\right.$ vs. scramble control)

\section{B}

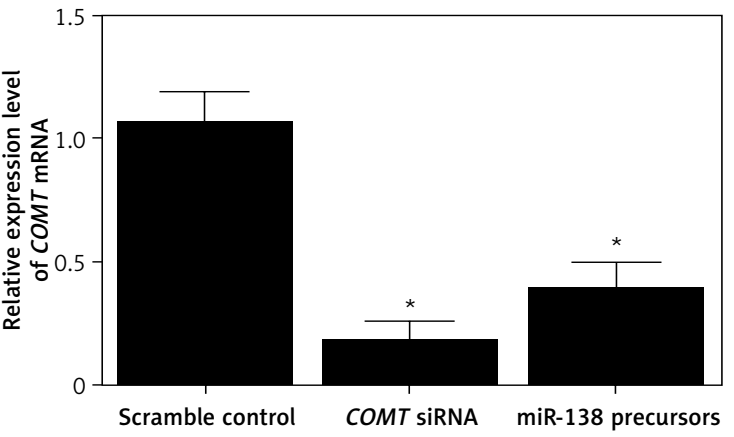

Figure 7. MiR-138 precursors slightly suppressed the expression of COMT in primary prostate cancer cells carrying the AA genotype. A - Dramatic elevation of miR-138 expression indicated efficient transfection of miR-138 precursors into primary prostate cancer cells carrying the AA genotype ( ${ }^{*} p<0.05$ vs. scramble control). B - The inhibition of COMT mRNA expression by COMT siRNA was stronger than that by miR-138 precursors in primary prostate cancer cells carrying the $A A$ genotype $\left({ }^{*} p<0.05\right.$ vs. scramble control). C - The inhibition of COMT protein expression by COMT siRNA was stronger than that by miR-138 precursors in primary prostate cancer cells carrying the AA genotype $\left({ }^{*} p<0.05\right.$ vs. scramble control) 
are associated with the risk of prostate cancer recurrence and the PSA-progression-free survival period. In this study, we found that the PSA-progression-free rate was highest in patients carrying the rs165599-AA + rs4680-Val/Val genotype and lowest in patients carrying the rs165599-(GG+GA) + rs4680- (Val/Met + Met/Met) genotype. In addition, the expression and activity of COMT were significantly higher in samples collected from patients carrying the rs165599-AA + rs4680-Val/ Val genotype, and they were remarkably lower in samples collected from patients carrying the rs165599-(GG+GA) + rs4680- (Val/Met + Met/ Met) genotype.

$\mathrm{Yu}$ et al. found that miR-138-5p displayed a suppressive impact on the growth of PC, suppressing the formation of tumours [30]. Tian et al. showed that miR-138-5p inhibited autography and PC growth, suggesting that miR-138-5p could function as a vital factor in PC [25, 32]. One SNP, rs 165599, demonstrated the highest correlation with mental illness [33]. While SNP rs165599 is actually located downstream of the site of COMT polyadenylation and hence cannot be found in a lot of EST sequences recorded in the GenBank database, this SNP could be amplified from brain cDNA free of genomic contamination, which confirms that SNP rs165599 is actually expressed in the brain [34]. As a result, the site of COMT with additional 3 polyadenylation should also be present in the human brain. When SNP rs 165599 was utilized as an expression tag for COMT transcripts containing the 3 sequence, notable variations were seen in allelic expression, indicating that the SNP is actually linked to larger modifications in the transcripts containing the 3' UTR. In this study, we carried out luciferase assays to explore the effect of rs 165599 polymorphisms on the expression of COMT. The luciferase activity of COMT-G vector was remarkably suppressed by miR-138 mimics. Furthermore, we cultured primary prostate cancer cells carrying GG and AA genotypes to explore the effect of miR-138 precursors on the expression of COMT. MiR-138 precursors showed a stronger effect on suppressing the expression of COMT mRNA and protein than COMT SiRNA in primary prostate cancer cells carrying the GG genotype, but not in primary prostate cancer cells carrying the AA genotype.

However, there are limitations to our study. The number of patients included in our study for genetic analysis was too small to be convincible and supportive. Also, the genetic analysis was not comprehensive. In our future study, it will be necessary to include a more comprehensive genetic analysis.

In conclusions, the findings of this study demonstrate that the carriers of the AA genotype of rs165599 and Val/Val genotype of rs4680 SNPs showed an increased risk of prostate cancer recurrence and shorter PSA-progression-free survival period, both of which indicated a worse prognosis. Therefore, these 2 polymorphisms may jointly function as a novel biomarker for the prognosis of prostate cancer recurrence.

\section{Acknowledgments}

This study was supported by Hubei Provincial Natural Science Foundation (No. 2018CFB786).

\section{Conflict of interest}

The authors declare no conflict of interest.

\section{References}

1. Jemal A, Siegel R, Xu J, Ward E. Cancer statistics, 2010. CA Cancer J Clin 2010; 60: 277-300.

2. Rethemiotaki I, Rethemiotakis A. Link between prostate cancer diagnosis and stroke in the United States during 2007-2017. Arch Med Sci Atheroscler Dis 2019; 4: 94-102.

3. Hirata K, Egawa S, Kimura Y, et al. Current status of surgery for pancreatic cancer. Dig Surg 2007; 24: 137-47.

4. Gillen S, Schuster T, Meyer Zum Buschenfelde C, Friess H, Kleeff J. Preoperative/neoadjuvant therapy in pancreatic cancer: a systematic review and meta-analysis of response and resection percentages. PLoS Med 2010; 7: e1000267.

5. Bastos P, Gomes T, Ribeiro L. Catechol-O-methyltransferase (COMT): an update on its role in cancer, neurological and cardiovascular diseases. Rev Physiol Biochem Pharmacol 2017; 173: 1-39.

6. Parkin GM, Udawela M, Gibbons A, Scarr E, Dean B. Catechol-O-methyltransferase (COMT) genotypes are associated with varying soluble, but not membrane-bound COMT protein in the human prefrontal cortex. J Hum Genet 2018; 63: 1251-8.

7. Matsuzaka CT, Christofolini D, Ota VK, et al. Catechol-O-methyltransferase (COMT) polymorphisms modulate working memory in individuals with schizophrenia and healthy controls. Braz J Psychiatry 2017; 39: 302-8.

8. Perkovic MN, Strac DS, Tudor L, Konjevod M, Erjavec GN, Pivac N. Catechol-O-methyltransferase, cognition and Alzheimer's disease. Curr Alzheimer Res 2018; 15: 408-19.

9. Zubenko GS, Maher BS, Hughes HB, 3rd, Zubenko WN, Scott Stiffler J, Marazita ML. Genome-wide linkage survey for genetic loci that affect the risk of suicide attempts in families with recurrent, early-onset, major depression. Am J Med Genet B Neuropsychiatr Genet 2004; 129B: 47-54.

10. Perlis RH, Huang J, Purcell $\mathrm{S}$, et al. Genome-wide association study of suicide attempts in mood disorder patients. Am J Psychiatry 2010; 167: 1499-507.

11. Nolan KA, Volavka J, Czobor P, et al. Suicidal behavior in patients with schizophrenia is related to COMT polymorphism. Psychiatr Genet 2000; 10: 117-24.

12. Baud P, Courtet P, Perroud N, Jollant F, Buresi C, Malafosse A. Catechol-O-methyltransferase polymorphism (COMT) in suicide attempters: a possible gender effect on anger traits. Am J Med Genet B Neuropsychiatr Genet 2007; 144B: 1042-7.

13. Pillai RS. MicroRNA function: multiple mechanisms for a tiny RNA? RNA 2005; 11: 1753-61. 
14. Calin GA, Croce CM. MicroRNA signatures in human cancers. Nat Rev Cancer 2006; 6: 857-66.

15. Feng W, Feng Y. MicroRNAs in neural cell development and brain diseases. Sci China Life Sci 2011; 54: 1103-12

16. Bian S, Sun T. Functions of noncoding RNAs in neural development and neurological diseases. Mol Neurobiol 2011; 44: 359-73.

17. Papagiannakopoulos T, Kosik KS. MicroRNAs: regulators of oncogenesis and stemness. BMC Med 2008; 6: 15.

18. Blandino G, Fazi F, Donzelli S, et al. Tumor suppressor microRNAs: a novel non-coding alliance against cancer. FEBS Lett 2014; 588: 2639-52.

19. Zhu Z, Tang J, Wang J, Duan G, Zhou L, Zhou X. MiR138 Acts as a Tumor Suppressor by Targeting EZH2 and Enhances Cisplatin-Induced Apoptosis in Osteosarcoma Cells. PLoS One 2016; 11: e0150026.

20. Jiang B, Mu W, Wang J, et al. MicroRNA-138 functions as a tumor suppressor in osteosarcoma by targeting differentiated embryonic chondrocyte gene 2. J Exp Clin Cancer Res 2016; 35: 69.

21. Xiao L, Zhou H, Li XP, et al. MicroRNA-138 acts as a tumor suppressor in non small cell lung cancer via targeting YAP1. Oncotarget 2016; 7: 40038-46.

22. Ye $X W, Y u H$, Jin $Y K$, et al. miR-138 inhibits proliferation by targeting 3-phosphoinositide-dependent protein kinase-1 in non-small cell lung cancer cells. Clin Respir J 2015; 9: 27-33.

23. Wang Q, Tang H, Yin S, Dong C. Downregulation of microRNA-138 enhances the proliferation, migration and invasion of cholangiocarcinoma cells through the upregulation of RhoC/p-ERK/MMP-2/MMP-9. Oncol Rep 2013; 29: 2046-52.

24. Song T, Zhang X, Wang C, et al. MiR-138 suppresses expression of hypoxia-inducible factor 1alpha (HIF-1alpha) in clear cell renal cell carcinoma 786-O cells. Asian Pac J Cancer Prev 2011; 12: 1307-11.

25. Tian S, Guo X, Yu C, Sun C, Jiang J. miR-138-5p suppresses autophagy in pancreatic cancer by targeting SIRT1. Oncotarget 2017; 8: 11071-82.

26. Suzuki M, Mamun MR, Hara K, et al. The Val158Met polymorphism of the catechol-O-methyltransferase gene is associated with the PSA-progression-free survival in prostate cancer patients treated with estramustine phosphate. Eur Urol 2005; 48: 752-9.

27. Ren X, Zhang L, Xiao Q, Huang D, Liu Q, Zhang Y. Association between COMT polymorphism, labor anxiety, and analgesia in pregnant women. J Pain Res 2019; 12: 779-85.

28. Lamb YN, Thompson JM, Murphy R, et al.; ABC Study group. Perceived stress during pregnancy and the catechol-O-methyltransferase (COMT) rs165599 polymorphism impacts on childhood IQ. Cognition 2014; 132: 461-70.

29. Szemraj-Rogucka Z, Szemraj J, Masiarek K, Majos A. Circulating microRNAs as biomarkers for myocardial fibrosis in patients with left ventricular non-compaction cardiomyopathy. Arch Med Sci 2019; 15: 376-84.

30. Yu C, Wang M, Li Z, et al. MicroRNA-138-5p regulates pancreatic cancer cell growth through targeting FOXC1. Cell Oncol (Dordr) 2015; 38: 173-81.

31. Brureau L, Moningo D, Emeville E, et al. Polymorphisms of estrogen metabolism-related genes and prostate cancer risk in two populations of African ancestry. PLoS One 2016; 11: e0153609.

32. Yang M, Sun S, Guo Y, Qin J, Liu G. Long non-coding RNA MCM3AP-AS1 promotes growth and migration through modulating FOXK1 by sponging miR-138-5p in pancreatic cancer. Mol Med 2019; 25: 55.
33. Shifman S, Bronstein $M$, Sternfeld $M$, et al. A highly significant association between a COMT haplotype and schizophrenia. Am J Hum Genet 2002; 71: 1296-302.

34. DeYoung CG, Getchell M, Koposov RA, et al. Variation in the catechol-O-methyltransferase Val 158 Met polymorphism associated with conduct disorder and ADHD symptoms, among adolescent male delinquents. Psychiatr Genet 2010; 20: 20-4. 